Article

\title{
Improving Eight-State Continuous Variable Quantum Key Distribution by Applying Photon Subtraction
}

\author{
Qingquan Peng ${ }^{1}{ }^{\infty}$, Xiaodong $\mathrm{Wu}^{1, *}$ and Ying Guo ${ }^{1,2}$ \\ 1 School of Automation, Central South University, Changsha 410083, China; weiyideai520@gmail.com (Q.P.); \\ yingguo@mail.csu.edu.cn (Y.G.) \\ 2 Jiangsu Key Construction Laboratory of IoT Application Technology, Wuxi Taihu University, Wuxi 214064, \\ China \\ * Correspondence: xiaodongwu@csu.edu.cn
}

Received: 28 January 2019; Accepted: 26 March 2019; Published: 29 March 2019

\begin{abstract}
We propose a new method to effectively improve the performance of a quantum key distribution with eight-state continuous variables by the photon subtraction method. This operation is effective in increasing and distilling Gaussian entanglement between quantum states, and can be easily realized by existing technology. Simulation results show that the channel-loss tolerance of the eight-state continuous variable quantum key distribution (CVQKD) protocol can be extended by the appropriate photon subtraction algorithm; namely, single-photon subtraction.
\end{abstract}

Keywords: quantum key distribution; eight-state continuous-variable protocol; photon subtraction

\section{Introduction}

Two independent long-distance legitimate communicators, say, Alice's station and Bob's station, can generate an unordered security key sequence through the untrusted quantum and traditional electronic channels by using quantum key distribution (QKD) [1,2]. The fundamental laws of quantum mechanics ensure their absolute security $[3,4]$. The traditional QKD protocol is usually divided into the following three categories: The discrete variable (DV) QKD protocol, which encodes information based on the polarization states of a single photon. The second is the continuous variable $(\mathrm{CV})$ QKD protocol [5-8], which encodes key bits in the quadrature of the optical field. The third is the distributed phase reference (DPR) QKD protocol; its main implementation is to use weak coherent pulses to pass information. Unlike DVQKD, in CVQKD there is no necessity to use a single photon detector. Additionally, the security of CVQKD against collective attacks $[9,10]$ and coherent attacks has been proved. Therefore, it can provide a more cost-effective solution, in practice, than its DVQKD counterpart [11]. While the DPRQKD protocol has been used in real settings for many years, it has never been completely secure [12].

Generally speaking, CVQKD can be mainly divided into two categories: Discrete modulation CVQKD [13] and Gaussian modulation CVQKD [14]. However, in the context of long-distance communication, in Gaussian modulation CVQKD it is difficult to harmonize the Gaussian variable required for modulation in the presence of a low SNR. Fortunately, there is no such limitation for discretely-modulated CVQKD protocols. It is worth noting that the current four-state CVQKD has been proven secure during an anti-collective attack, which has been experimentally demonstrated [15-17]. Recently, another discretely-modulated scheme, called the eight-state CVQKD, has been proposed, which shows better performance compared with the four-state CVQKD $[18,19]$.

Non-Gaussian operations (including photon addition and photon subtraction) have been diffusely studied in both theory and experiments. By taking advantage of these operations, the entanglement of an Gaussian entangled state is improved and distilled, and thus the maximum secure distance is 
well-enhanced [20-22]. The implementation of a photon subtraction operation is simple, as it can be smoothly performed with optical beam splitters and photon detectors. In this paper, we deployed a photon subtraction operation on Alice's side, in order to effectively improve the performance of the eight-state protocol. A security analysis shows that the performance of the eight-state protocol can be improved with proper photon subtraction.

The structure of this paper is as follows. In Section 2, we introduce the original eight-state CVQKD protocol, and then introduce the modified protocol (applying a photon-subtraction operation). In Section 3, we perform a security analysis of the modified protocol. Simulation results are illustrated in Section 4. Finally, the conclusion is drawn in Section 5.

\section{Eight-State CVQKD with Photon Subtraction Operation}

This section is divided into two parts. The original eight-state CVQKD protocol is first explained. Then, we introduce a modified eight-state protocol, based on the entanglement-based (EB) scheme.

\subsection{Eight-State CVQKD Protocol}

In the eight-state protocol, the eight coherent displaced states $\left|\alpha_{k}^{8}\right\rangle$ prepared by Alice are given by

$$
\left|\alpha_{k}^{8}\right\rangle=\left|\alpha e^{\frac{i k \pi}{4}}\right\rangle,
$$

where $k$ is the number of coherent displacement states (ranging from 0 to 7 ), and $\alpha$ is a positive real number obtained from the modulation variance of the coherent state. After that, Alice sends one state to Bob, the state being randomly obtained from eight states. When this state reaches Bob's station by the quantum channel, Bob measures it using a homodyne or heterodyne detector.

Bob's received noise variance can be expressed as $(1+\tau \epsilon) N_{0}$. This noise is mainly generated by the interaction of the excess noise $\epsilon$ and the transmission efficiency $\tau$, where $N_{0}$ is expressed as the shot-noise limit. Therefore, the total noise received at the Bob's station can be given by $X_{\text {line }}=1 / \tau+\epsilon-1$ [18]. Finally, they achieve the final secret key by taking advantage of privacy amplification and error correction. In the EB protocol, Alice needs to prepare a pure two-mode quantum state. The received matrix $\rho_{8}$ can be expressed as

$$
\begin{aligned}
\rho_{8} & =\frac{1}{8} \sum_{k=0}^{7}\left|\alpha_{k}^{8}\right\rangle\left\langle\alpha_{k}^{8}\right| \\
& =\sum_{k=0}^{7} \lambda_{k}\left|\phi_{k}\right\rangle\left\langle\phi_{k}\right|,
\end{aligned}
$$

where

$$
\begin{aligned}
& \lambda_{0(4)}=\frac{1}{4} e^{-\alpha^{2}}\left[\cosh \left(\alpha^{2}\right)+\cos \left(\alpha^{2}\right) \pm 2 \cos \left(\frac{\alpha^{2}}{\sqrt{2}}\right) \cosh \left(\frac{\alpha^{2}}{\sqrt{2}}\right)\right], \\
& \lambda_{1(5)}=\frac{1}{4} e^{-\alpha^{2}}\left[\sinh \left(\alpha^{2}\right)+\sin \left(\alpha^{2}\right) \pm \sqrt{2} \cos \left(\frac{\alpha^{2}}{\sqrt{2}}\right) \sinh \left(\frac{\alpha^{2}}{\sqrt{2}}\right) \pm \sqrt{2} \sin \left(\frac{\alpha^{2}}{\sqrt{2}}\right) \cosh \left(\frac{\alpha^{2}}{\sqrt{2}}\right)\right], \\
& \lambda_{2(6)}=\frac{1}{4} e^{-\alpha^{2}}\left[\sinh \left(\alpha^{2}\right)-\sin \left(\alpha^{2}\right) \pm 2 \sin \left(\frac{\alpha^{2}}{\sqrt{2}}\right) \sinh \left(\frac{\alpha^{2}}{\sqrt{2}}\right)\right], \\
& \lambda_{3(7)}=\frac{1}{4} e^{-\alpha^{2}}\left[\sinh \left(\alpha^{2}\right)-\sin \left(\alpha^{2}\right) \mp \sqrt{2} \cos \left(\frac{\alpha^{2}}{\sqrt{2}}\right) \sinh \left(\frac{\alpha^{2}}{\sqrt{2}}\right) \pm \sqrt{2} \sin \left(\frac{\alpha^{2}}{\sqrt{2}}\right) \cosh \left(\frac{\alpha^{2}}{\sqrt{2}}\right)\right],
\end{aligned}
$$

and

$$
\left|\phi_{k}\right\rangle=\frac{e^{-\alpha^{2} / 2}}{\sqrt{\lambda_{k}}} \sum_{n=0}^{\infty} e \frac{\alpha^{8 n+k}}{\sqrt{(8 n+k) !}}|8 n+k\rangle, k \in\{0,1,2 \ldots, 7\} .
$$


When we apply the annihilation operator $\hat{\alpha}$ to $\left|\phi_{k}\right\rangle$, we obtain $\hat{\alpha}\left|\phi_{0}\right\rangle=-\alpha \sqrt{\lambda_{7}} / \sqrt{\lambda_{0}}\left|\phi_{7}\right\rangle$, and $\hat{\alpha}\left|\phi_{k}\right\rangle=\alpha \sqrt{\lambda_{k-1}} / \sqrt{\lambda_{k}}\left|\phi_{k-1}\right\rangle, k \in\{0,1,2 \ldots, 7\}$. Therefore, the state $\rho_{8}$ can be further processed as

$$
\begin{aligned}
\left|\Psi_{8}\right\rangle & =\sum_{k=0}^{7} \sqrt{\lambda_{k}}\left|\phi_{k}\right\rangle\left|\phi_{k}\right\rangle \\
& =\frac{1}{4} \sum_{k=0}^{7}\left|\psi_{k}\right\rangle\left|\alpha_{k}^{8}\right\rangle,
\end{aligned}
$$

where the states $\left|\psi_{k}\right\rangle$ are the orthogonal non-Gaussian states

$$
\left|\psi_{k}\right\rangle=\frac{1}{2} \sum_{m=0}^{7} e^{i(4 k+1) m \pi}\left|\phi_{m}\right\rangle, k \in\{0,1,2 \ldots, 7\}
$$

Among these, Alice prepares the quantum entangled states $\left|\psi_{k}\right\rangle\left\langle\psi_{k}\right|(k \in\{0,1,2 \ldots, 7\})$ and performs projection measurements, one of which enters the first half of $\left|\Psi_{8}\right\rangle$, while the other half is projected to the eight coherent states $\left|\phi_{k}\right\rangle(k \in\{0,1,2 \ldots, 7\})$ with equal probability.

Now, we analyze the covariance matrix $\gamma_{8}$ of state $\left|\Psi_{8}\right\rangle$, which is given by

$$
\gamma_{8}=\left[\begin{array}{cc}
X L_{2} & Z_{8} \sigma_{z} \\
Z_{8} \sigma_{z} & Y L_{2}
\end{array}\right],
$$

where the $L_{2}$ represents a diagonal matrix of $[1,1], \sigma_{z}$ represents a diagonal matrix of $[1,-1]$, and

$$
\begin{gathered}
X=\left\langle\Psi_{8}\left|2 a^{\dagger}+1\right| \Psi_{8}\right\rangle, \\
Y=\left\langle\Psi_{8}\left|2 b^{\dagger}+1\right| \Psi_{8}\right\rangle, \\
Z_{8}=\left\langle\Psi_{8}\left|a b+a^{\dagger} b^{\dagger}\right| \Psi_{8}\right\rangle,
\end{gathered}
$$

where $a, b, a^{\dagger}$, and $b^{\dagger}$ are operators associated with the Alice and Bob modes, respectively; $a$ and $a^{\dagger}$ are the annihilation operators, and $b$ and $b^{\dagger}$ are the creation operators. Thus, $X, Y$, and $Z_{8}$ in the covariance matrix $\gamma_{8}$ can be calculated as

$$
\begin{aligned}
X & =Y=2 a^{2}+1, \\
Z_{8} & =2 a^{2} \sum_{k=0}^{7} \lambda_{k-1}^{\frac{3}{2}} \lambda_{k}^{-\frac{1}{2}} .
\end{aligned}
$$

In addition, $V_{a}=2 a^{2}$.

Afterwards, Bob obtains a final key, shared with Alice by the implementation of reverse reconciliation and a privacy amplifier.

\subsection{Eight-State $C V Q K D$ with Photon Subtraction Operation}

In what follows, as shown in Figure 1, we suggest an improved eight-state CVQKD scheme by using the photon-subtraction operation. Here, Alice takes advantage of a beam splitter (BS) to split modes $B$ and $C_{0}$ (vacuum state) into mode $B_{1}$ and mode $C$, where the states $A, C$, and $B_{1}$ can be expressed as

$$
\rho_{A C B_{1}}=U_{B S}\left[\left|\Psi_{8}\right\rangle\left\langle\Psi_{8}|\otimes| 0\right\rangle\langle 0|\right] U_{B S}^{+} .
$$

For the measurement of mode $C$, Alice gets $\left\{\widehat{\Pi}_{0}, \widehat{\Pi}_{1}\right\}$ by utilizing positive operator-valued measurement (POVM) in the photon-number-resolving detector (PNRD). The number of photon 
reductions is related to $\widehat{\Pi}_{1}=|k\rangle\langle k|$. The modes $A$ and $B_{1}$ are kept by Alice and Bob when the POVM element $\widehat{\Pi}_{1}$ clicks. The photon-subtracted state $\rho_{A B_{1}}^{\widehat{\Pi}_{1}}$ is expressed by

$$
\rho_{A B_{1}}^{\widehat{\Pi}_{1}}=\frac{\operatorname{tr}_{C}\left(\widehat{\Pi}_{1} \rho_{A C B_{1}}\right)}{\operatorname{tr}_{A C B_{1}}\left(\widehat{\Pi}_{1} \rho_{A C B_{1}}\right)}
$$

where $\operatorname{tr}_{X}(\cdot)$ is a multimode quantum state part. Our success probability of subtracting $k$ photons is denoted by $P_{(k)}^{\widehat{\Pi}_{1}}$, which is given by

$$
\begin{aligned}
P_{(k)}^{\widehat{\Pi}_{1}} & =\operatorname{tr}_{A C B_{1}}\left(\widehat{\Pi}_{1} \rho_{A C B_{1}}\right) \\
& =\left(1-\lambda^{2}\right) \sum_{n=k}^{\infty} \lambda^{2 n} C_{n}^{k} \mu^{n-k}(1-\mu)^{k} \\
& =\frac{1-\lambda^{2}}{1-\mu \lambda^{2}}\left[\frac{\lambda^{2}(1-\mu)}{1-\mu \lambda^{2}}\right]^{k},
\end{aligned}
$$

where $\lambda=\sqrt{(V-1) /(V+1)}$. It is worth noting that the state $\rho_{A B_{1}}^{\widehat{\Pi}_{1}}$ is no longer a Gaussian distribution after the photon-subtraction operation. Here, Alice performs heterodyne detection. Note that it can be converted into an electrical signal without distortion by selecting a suitable photodetector. When the circuit completes the inspection task, the photodetector ultimately detects the required information in the form of an output electrical signal, which helps to generate a key sequence when interacting with Bob. Bob can perform heterodyne or homodyne detection. In this experiment, we focus our attention on the homodyne measurement. Suppose $\gamma_{8}^{(k)}$ represents the covariance matrix of $\rho_{A B_{2}}^{\widehat{\Pi}_{1}}$, which is expressed as ([20]):

$$
\gamma_{8}^{(k)}=\left[\begin{array}{ll}
X^{\prime} L_{2} & Z_{8}^{\prime} \sigma_{z} \\
Z_{8}^{\prime} \sigma_{z} & Y^{\prime} L_{2}
\end{array}\right],
$$

where

$$
\begin{aligned}
& X^{\prime}=\frac{\mu \lambda^{2}+2 k+1}{1-\mu \lambda^{2}}, \\
& Y^{\prime}=\frac{\mu \lambda^{2}(2 k+1)+1}{1-\mu \lambda^{2}}, \\
& Z_{8}^{\prime}=\frac{2 \sqrt{\mu} \lambda(k+1)}{1-\mu \lambda^{2}} .
\end{aligned}
$$

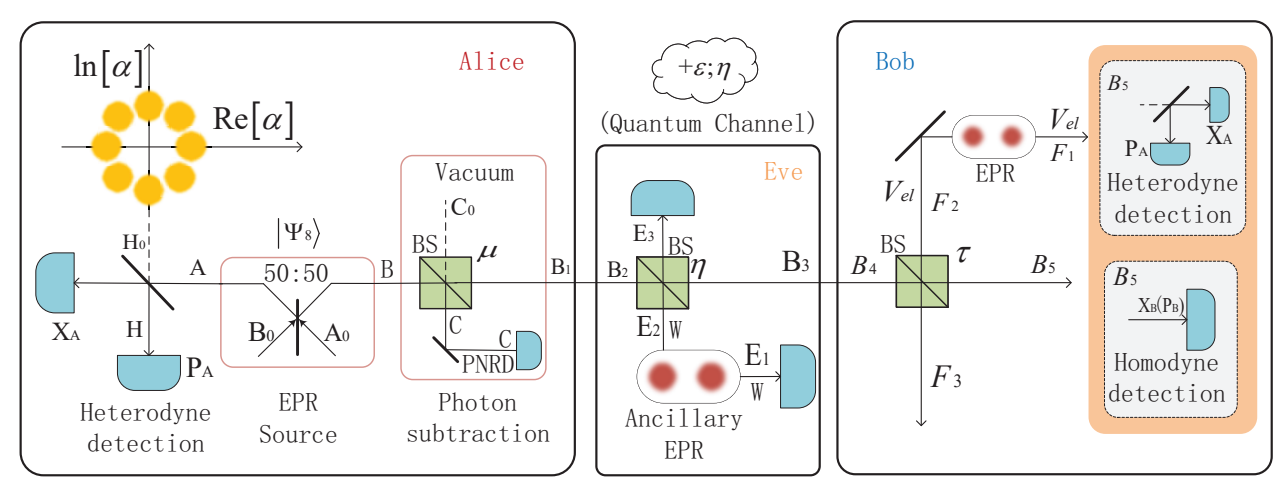

Figure 1. Prototype diagram of an improved eight-state discrete-modulated continuous variable quantum key distribution (CVQKD). Alice prepares one of the eight-state discrete modulation states. The rest of the state is passed through the photon subtraction module and then passed through the untrusted quantum channel to Bob. Bob will use homodyne or heterodyne detection to derive the key sequence shared by Alice. 


\section{Security Analysis}

\subsection{The Optimal Value}

We need to find the optimal value for $V_{a}$, because this value will directly determine whether the performance of the eight-state CVQKD protocol shows the best performance. First, we use the content in Section 2 to build a data model of the original eight-state protocol. We keep the other parameters unchanged in the data model, but only change the transmission distance parameter $d$ (excess noise $\epsilon$ is fixed to legitimate value), and establish a relationship diagram between $V_{a}$ and the secret key rate. As shown in Figure 2, we find that the secret key rate achieves the highest value when $V_{a}=0.25$.

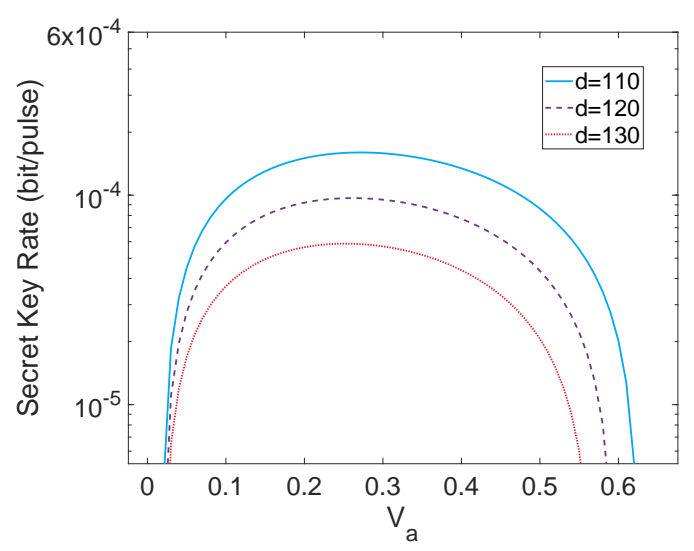

Figure 2. When $\epsilon=0.01$, the relationship between $V_{a}$ and secret key rate with different values of $d$.

We need to further verify the $V_{a}$ value. The transmission distance $d$ is fixed to a legitimate value and the other parameters are kept unchanged. Here, we choose different values of the excess noise $\epsilon$. From Figure 3, we observe that the optimal $V_{a}$ value still exists in a public interval including 0.25 .

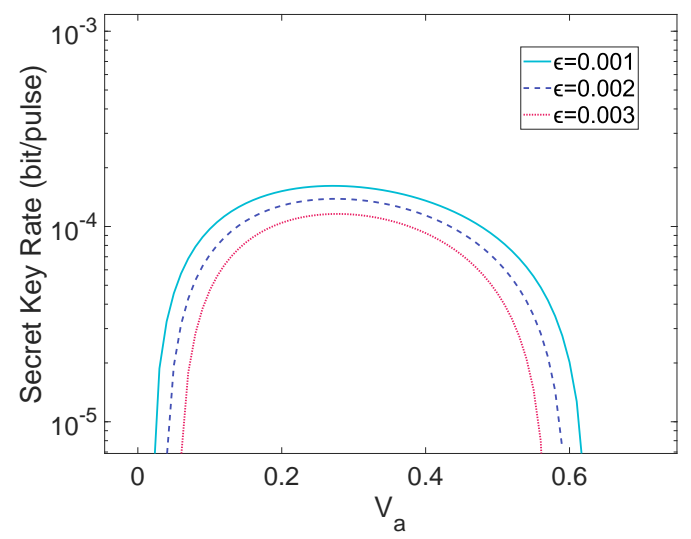

Figure 3. When $d=110 \mathrm{~km}$, the relationship between $V_{a}$ and secret key rate with different values of $\epsilon$.

Therefore, we take the value of $V_{a}$ to be 0.25 in the following analysis.

\subsection{Secret Key Rate}

In this section, we calculate the secure key rate of the modified protocol. In this case, we performed heterodyne detection and homodyne detection with Alice and Bob, respectively. While Eve only performs a collective attack to get the best results, and the information obtained is limited to the Holevo range $S(E: B)$. It should be noted that the state $\rho_{A B_{1}}^{\widehat{\Pi}_{1}}$ is no longer a Gaussian state after the photon subtraction operation is performed. Therefore, here, we cannot directly apply the traditional Gaussian CVQKD results to calculate the secret key rate. We derive the secure key rate based on the 
extremum of the Gaussian quantum states and the corresponding object with the same covariance matrix

$$
K=P_{(k)}^{\widehat{\Pi}_{1}}[\beta I(A: B)-S(E: B)],
$$

where $I(A: B)$ and $S(E: B)$ correspond to the mutual information between Alice-Bob and Eve-Bob, and $\beta$ is the efficiency of reverse reconciliation. When the state emitted by Alice passes through the channel controlled by Eve, the covariance matrix $\gamma_{A_{3}}$ is expressed as

$$
\gamma_{A B_{3}}=\left[\begin{array}{ll}
a L_{2} & c \sigma_{z} \\
c \sigma_{z} & b L_{2}
\end{array}\right],
$$

where $a=X^{\prime}, b=\eta\left(Y^{\prime}+X_{\text {line }}\right)$, and $c=\sqrt{\eta} Z_{8}^{\prime}$.

Then, $I(A: B)$ is derived based on Bob's measurement, which is calculated by

$$
I(A: B)=\frac{1}{2} \log _{2} \frac{V_{A}}{V_{A \mid B}},
$$

with $V_{A}=(a+1) / 2$ and $V_{B}=b$ are the respective variances, and $V_{A \mid B}$ is the conditional variance, namely

$$
V_{A \mid B}=V_{A}-\frac{\eta Z_{8}^{\prime}}{2 V_{B}}=a-\frac{c^{2}}{2 b}
$$

Eve can purify the system to interact with Bob after Bob performs homodyne detection. Based on this, the Holevo bound is given by

$$
\begin{aligned}
S(E: B) & =S(E)-S(E \mid B) \\
& =G\left[\left(\lambda_{1}-1\right) / 2\right]+G\left[\left(\lambda_{2}-1\right) / 2\right]-G\left[\left(\lambda_{3}-1\right) / 2\right],
\end{aligned}
$$

where

$$
G(x)=(x+1) \log _{2}(x+1)-x \log _{2} x,
$$

and the eigenvalues, $\lambda_{1,2,3}$, in the equation can be calculated by

$$
\begin{aligned}
\lambda_{1,2}^{2} & =\frac{1}{2}\left(A \pm \sqrt{A^{2}-4 B}\right), \\
\lambda_{3}^{2} & =\frac{a^{2}-a c^{2}}{b},
\end{aligned}
$$

with $A=a^{2}+b^{2}-2 c^{2}$ and $B=a b-c^{2}$.

\section{Performance and Discussion}

In Figure 4, based on the improved eight-state CVQKD protocol, we show the relationship between the secret key rate and the transmission distance of the protocol. The left-to-right relationship curves correspond to the original eight-state protocol, three-photon subtraction, two-photon subtraction, and one-photon subtraction. We compare the original eight-state protocol and the modified protocol. Interestingly, the less photons we subtract, the better performance we can achieve. Therefore, we obtain optimal performance of the eight-state protocol by performing a one-photon subtraction operation.

Note that in actual use, the noise $\epsilon$ often exists in the quantum channel. Therefore, studying $\epsilon$ is very important for the performance of our improved eight-state CVQKD protocol. The relationship between the secret key rate and channel loss is shown in Figure 5. The left-to-right relationship curves correspond to $\epsilon=0.032, \epsilon=0.025, \epsilon=0.008$, and $\epsilon=0.001$, respectively. We find that the use of a photon-subtraction operation can tolerate more channel loss. 


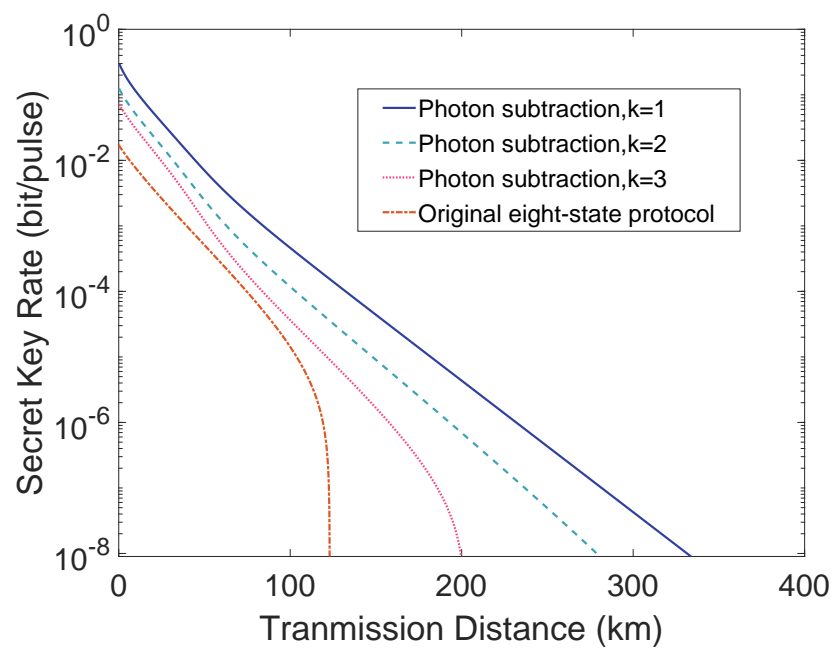

Figure 4. Performance of eight-state protocol with a photon subtraction operation. Here, $\epsilon=0.001$ and $\beta=0.95$. The orange line is for the original eight-state protocol and the other lines are for the photon subtraction with $k \in\{1,2,3\}$, respectively.

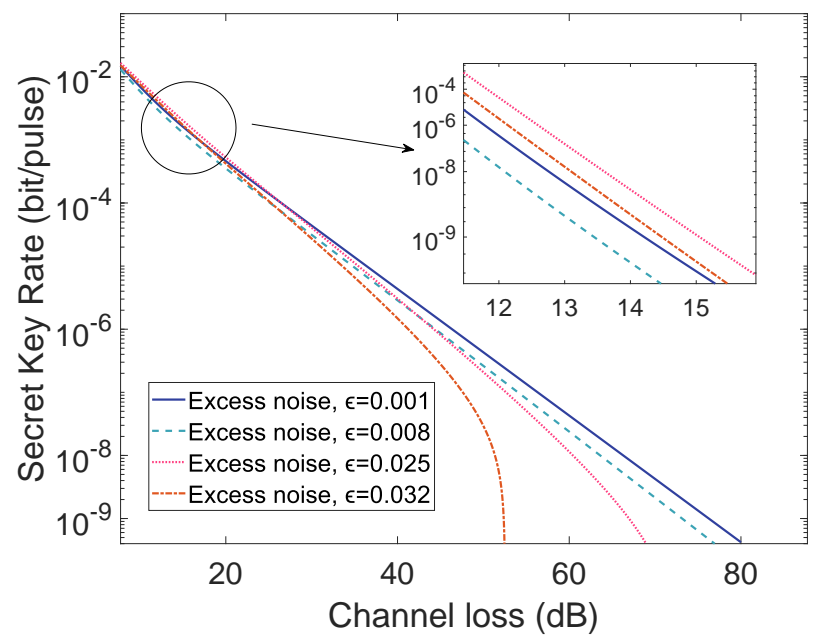

Figure 5. The relationship between the secret key rate of the system and different values of the excess noise $\epsilon$ under the one-photon subtraction operation of the eight-state CVQKD.

As the photon-subtraction operation is utilized on Alice's side, the intensity of the light field is improved and, thus, the entanglement in the EPR states generated by Alice can be well-enhanced, which ultimately contributes to the performance improvement (tolerating more channel loss) of the eight-state protocol, as illustrated in simulation results above.

\section{Conclusions}

This paper presents a method for enhancing the performance of eight-state CVQKD, by use of photon subtraction on the original eight-state CVQKD. Only using optical beam splitters and photon detectors, this operation can be well-applied in practice. The simulation results show that the eight-state CVQKD with photon subtraction significantly improved the transmission distance. In addition, it is very important to control the excess noise, in practice, since this noise has a significant impact on the performance of the proposed protocol. It is worth noting that the results also show that the optimal performance can be obtained by utilizing a one-photon subtraction operation. 
Author Contributions: X.W. gave a general idea of the study, designed the concept of the study, and provided relevant studies and literature. Q.P. wrote the article and completed the derivation of formulae and simulation analysis. Y.G. provided practical recommendations for this study. X.W. revised some of the content in the paper. All authors have read and approved the final manuscript.

Funding: This work is supported by the National Natural Science Foundation of China (Grant No. 61379153, No. 61572529).

Acknowledgments: This work was supported by the National Natural Science Foundation of China (Grant Nos. 61572529, 61871407, 61872390, 61801522), and the Natural Science Foundation of the Jiangsu Higher Education Institutions of China (Grant No. 18KJB510045).

Conflicts of Interest: The authors declare no conflict of interest.

\section{References}

1. Gisin, N.; Ribordy, G.; Tittel, W.; Zbinden, H. Quantum cryptography. Rev. Mod. Phys. 2002, 74, 145. [CrossRef]

2. Scarani, V.; Bechmann-Pasquinucci, H.; Cerf, N.J.; Dušek, M.; Lütkenhaus, N.; Peev, M. The security of practical quantum key distribution. Rev. Mod. Phys 2009, 81, 1301. [CrossRef]

3. Wootters, W.K.; Zurek, W.H. A single quantum cannot be cloned. Nature 1982, 299, 802-803. [CrossRef]

4. Bang, J.Y.; Berger, M.S. Quantum mechanics and the generalized uncertainty principle. Phys. Rev. D 2006, 74, 125012. [CrossRef]

5. Ma, X.C.; Sun, S.H.; Jiang, M.S.; Gui, M.; Liang, L.M. Gaussian-modulated coherent-state measurement-device-independent quantum key distribution. Phys. Rev. A 2014, 89, 042335. [CrossRef]

6. Lance, A.M.; Symul, T.; Sharma, V.; Weedbrook, C.; Ralph, T.C.; Lam, P.K. No-switching quantum key distribution using broadband modulated coherent light. Phys. Rev. Lett. 2005, 95, 180503. [CrossRef] [PubMed]

7. Huang, P.; Fang, J.; Zeng, G. State-discrimination attack on discretely modulated continuous-variable quantum key distribution. Phys. Rev. A 2014, 89, 042330. [CrossRef]

8. Weedbrook, C.; Pirandola, S.; García-Patrón, R.; Cerf, N.J.; Ralph, T.C.; Shapiro, J.H.; Lloyd, S. Gaussian quantum information. Rev. Mod. Phys. 2012, 84, 621. [CrossRef]

9. Inamori, H.; Lütkenhaus, N.; Mayers, D. Unconditional security of practical quantum key distribution. Eur. Phys. J. D 2007, 41, 599. [CrossRef]

10. Grosshans, F.; Grangier, P. Continuous variable quantum cryptography using coherent states. Phys. Rev. Lett. 2002, 88, 057902. [CrossRef]

11. Weedbrook, C.; Lance, A.M.; Bowen, W.P.; Symul, T.; Ralph, T.C.; Lam, P.K. Quantum cryptography without switching. Phys. Rev. Lett 2004, 93, 170504. [CrossRef] [PubMed]

12. Moroder, T.; Curty, M.; Lim, C.C.W.; Zbinden, H.; Gisin, N. Security of distributed-phase-reference quantum key distribution. Phys. Rev. Lett. 2012, 109, 260501. [CrossRef] [PubMed]

13. Zhang, H.; Fang, J.; He, G. Improving the performance of the four-state continuous-variable quantum key distribution by using optical amplifiers. Phys. Rev. A 2012, 86, 022338. [CrossRef]

14. Pirandola, S.; Ottaviani, C.; Spedalieri, G.; Weedbrook, C.; Braunstein, S.L.; Lloyd, S.; Gehring, T.; Jacobsen, C.S.; Andersen, U.L. High-rate measurement-device-independent quantum cryptography. Nat. Photonics 2015, 9, 397. [CrossRef]

15. Zhou, J.; Huang, D.; Guo, Y. Long-distance continuous-variable quantum key distribution using separable Gaussian states. Phys. Rev. A 2018, 98, 042303. doi/10.1103/PhysRevA.98.042303. [CrossRef]

16. Li, F.; Wang, Y.; Liao, Q.; Guo, Y. Four-State Continuous-Variable Quantum Key Distribution with Photon Subtraction. Int. J. Theor. Phys 2018, 1-12. [CrossRef]

17. Wu, X.D.; Liao, Q.; Huang, D.; Wu, X.H.; Guo, Y. Balancing four-state continuous-variable quantum key distribution with linear optics cloning machine. Chin. Phys. B 2017, 26, 110304. [CrossRef]

18. Guo, Y.; Li, R.; Liao, Q.; Zhou, J.; Huang, D. Performance improvement of eight-state continuous-variable quantum key distribution with an optical amplifier. Phys. Lett. A 2018, 382, 372-381. [CrossRef]

19. Zhang, H.; Mao, Y.; Huang, D.; Guo, Y.; Wu, X.; Zhang, L. Finite-size analysis of eight-state continuous-variable quantum key distribution with the linear optics cloning machine. Chin. Phys. B 2018, 27, 090307. [CrossRef] 
20. Li, Z.; Zhang, Y.; Wang, X.; Xu, B.; Peng, X.; Guo, H. Non-Gaussian postselection and virtual photon subtraction in continuous-variable quantum key distribution. Phys. Rev. A 2016, 93, 012310. [CrossRef]

21. Guo, Y.; Liao, Q.; Wang, Y.; Huang, D.; Huang, P.; Zeng, G. Performance improvement of continuous-variable quantum key distribution with an entangled source in the middle via photon subtraction. Phys. Rev. A 2017, 95, 032304. [CrossRef]

22. Huang, P.; He, G.; Fang, J.; Zeng, G. Performance improvement of continuous-variable quantum key distribution via photon subtraction. Phys. Rev. A 2013, 87, 012317. [CrossRef]

(C) 2019 by the authors. Licensee MDPI, Basel, Switzerland. This article is an open access article distributed under the terms and conditions of the Creative Commons Attribution (CC BY) license (http:/ / creativecommons.org/licenses/by/4.0/). 